\title{
Search for supersymmetry in events with $b$ jets and missing transverse momentum at the LHC
}

\section{The CMS Collaboration}

ABSTRACT: A search for supersymmetry is presented using a sample of events with $b$ jets and missing transverse momentum. The search uses a data sample of proton-proton collisions at a centre-of-mass energy of $7 \mathrm{TeV}$, corresponding to an integrated luminosity of $35 \mathrm{pb}^{-1}$, collected with the CMS detector. A total of $0.33_{-0.33}^{+0.43}$ (stat.) \pm 0.13 (syst.) events is predicted, using control samples in the data, to arise from standard model processes, and one event is observed in the data. Upper limits are set at the $95 \%$ confidence level on the cross sections of benchmark supersymmetric models.

KEYWORDS: Hadron-Hadron Scattering 


\section{Contents}

1 Introduction 1

2 Event selection $\quad 2$

3 Background estimation 3

3.1 Background prediction using $\alpha_{\mathrm{T}}$ vs $H_{\mathrm{T}}$ extrapolation 3

3.2 Cross-checks of $\mathrm{Z} \rightarrow \nu \bar{\nu}$ and $t \bar{t}$ background contributions 4

4 Signal selection efficiency 5

5 Results $\quad 6$

$\begin{array}{lll}6 & \text { Summary } & 7\end{array}$

$\begin{array}{ll}\text { The CMS collaboration } & 10\end{array}$

\section{Introduction}

Supersymmetry (SUSY) [1-5] is an extension of the standard model (SM) of particle physics, which can solve the "hierarchy problem" $[6,7]$ and provide a candidate for cold dark matter [8]. For a large class of supersymmetric parameter sets, squarks ( $\tilde{\mathrm{q}})$, the SUSY partners of quarks, are relatively light. In this case, significant event yields at the Large Hadron Collider (LHC) can result from strong production of squarks, which subsequently decay giving a weakly interacting lightest supersymmetric particle (LSP). If bottom and top squarks, which can decay to b quarks, are relatively light, there may be an abundance of events with one or more b-quark jets and momentum imbalance transverse to the beam line due to the undetectable LSPs.

This Letter describes a search for events with two or more hadronic jets, at least one of which must be b tagged [9], and significant transverse momentum imbalance. It extends a similar search without a b-tag requirement [10]. The momentum imbalance is characterized [11] by the ratio of the $p_{\mathrm{T}}$ of the second-highest- $p_{\mathrm{T}}$ jet and the invariant mass formed from the two highest- $p_{\mathrm{T}}$ jets. This ratio can be estimated by $\alpha_{\mathrm{T}}=\frac{1}{2} \frac{H_{\mathrm{T}}-\Delta H_{\mathrm{T}}}{\sqrt{H_{\mathrm{T}}^{2}-H_{\mathrm{T}}^{2}}}$, where $H_{\mathrm{T}}=\left|\sum_{i} \vec{p}_{\mathrm{T}}^{\text {jet }_{i}}\right|, H_{\mathrm{T}}=\sum_{i} p_{\mathrm{T}}^{\text {jet }_{i}}$, and $p_{\mathrm{T}}^{\text {jet }_{i}}$ is the momentum transverse to the beam line for jet $i$ in an event. The jets in an event are grouped into two pseudo-jets and $\Delta H_{\mathrm{T}}$ is the minimal value of $\left|p_{\mathrm{T}}^{\text {pseudojet1 }}-p_{\mathrm{T}}^{\text {pseudojet2 }}\right|$ over all combinations; this approach optimizes rejection of backgrounds with apparent $H_{\mathrm{T}}$ from instrumental effects and other sources.

The main backgrounds are due to standard model multijet production (hereafter denoted "QCD background"), electroweak W and Z boson production (EWK), and top quark 
pair production $(t \bar{t})$. Owing to low average $H_{\mathrm{T}}$, the QCD background is effectively rejected by a requirement on $\alpha_{\mathrm{T}}$. The b-tag requirement further suppresses the QCD and EWK backgrounds.

The results of the search are characterized in terms of the mSUGRA/CMSSM $[12,13]$ scenario of SUSY. These models are described by four parameters and one sign: the universal scalar and gaugino mass parameters, $m_{0}$ and $m_{1 / 2}$, respectively; the universal trilinear coupling, $A_{0}$; the ratio of the two Higgs doublet vacuum expectation values, $\tan \beta$; and the sign of the Higgs mixing parameter, $\operatorname{sign}(\mu)$. Three signal points are considered as benchmarks: LM0, LM1, both discussed in ref. [10], and LMB (corresponding to $m_{0}=400 \mathrm{GeV}$, $m_{1 / 2}=200 \mathrm{GeV}, A_{0}=0 \mathrm{GeV}, \tan \beta=50$, and $\left.\operatorname{sign}(\mu)>0\right)$, chosen to be near the edge of sensitivity of this search in mSUGRA/CMSSM parameter space.

The choice of $\tan \beta=50$ for the LMB point is appropriate because of its b-quark enrichment. However, this choice of parameter space is merely a benchmark; light bottom and top squarks are in fact a generic feature of many supersymmetiric models. This analysis extends the sensitivity beyond ref. [10] for such b-quark enriched models because of the reduced backgrounds.

The analysis presented here uses a data sample of proton-proton collisions at $7 \mathrm{TeV}$, corresponding to an integrated luminosity of $35 \mathrm{pb}^{-1}$, collected with the Compact Muon Solenoid (CMS) detector, at the LHC. The main components of CMS are a silicon pixel and strip tracker, the crystal electromagnetic calorimeter, and the brass/scintillator hadron calorimeter, all placed in a $3.8 \mathrm{~T}$ axial magnetic field, complemented by gas-ionization detectors embedded in the steel return yoke, to measure muons. A detailed description of the detector and its performance can be found in ref. [14]. In the cylindrical coordinate system of CMS, $\phi$ is the azimuthal angle and the pseudo-rapidity $(\eta)$ is defined as $\eta=-\ln [\tan (\theta / 2)]$, where $\theta$ is the polar angle with respect to the counterclockwise beam direction.

\section{Event selection}

The event selection requirements are mostly identical to those in ref. [10]. Events in the search sample are collected with triggers based on $H_{\mathrm{T}}$ computed from jets reconstructed at trigger level. A muon-enriched control sample is collected with triggers requiring a muon. Events must have a good reconstructed pp collision vertex [15]. Jets are reconstructed as clusters of energy in the calorimeters by the anti- $\mathrm{k}_{\mathrm{T}}$ algorithm [16] with a distance parameter of 0.5 , and are required to have energy transverse to the beam, $E_{\mathrm{T}}$, in excess of $50 \mathrm{GeV}$ and $|\eta|$ less than 3 .

To perform a fully hadronic final state search and to reduce the backgrounds, events with an isolated lepton (electron or muon) or photon are vetoed, and events consistent with having apparent $H_{T}[10]$ are rejected. Selected events are required to have at least two jets, both with $E_{\mathrm{T}}>100 \mathrm{GeV},|\eta|<2.5$ for the highest- $E_{\mathrm{T}}$ jet, $H_{\mathrm{T}}>350 \mathrm{GeV}$, at least one jet tagged as originating from a $\mathrm{b}$ quark, and $\alpha_{\mathrm{T}}>0.55$.

Jets are $\mathrm{b}$ tagged using a discriminator based on the impact parameter significance of tracks in a jet (Track Counting High Purity discriminator, TCHP [9]), with a "tight" selection (TCHP > 3.41) designed to have a light-flavour contamination of less than $0.1 \%$. 
Looser b-tagging selections are used to produce various control samples. An event is said to be anti-tagged if it contains no jets with a loose $\mathrm{b}$ tag (TCHP $>1.19)$. To remain within the acceptance of the pixel tracker, only jets with a central axis of $|\eta|<2.4$ are considered for $\mathrm{b}$ tagging.

\section{Background estimation}

The backgrounds for this search can be categorized into three main groups: namely QCD, EWK, and $t \bar{t}$. The contamination from $t \bar{t}$ is mainly in the tau decay mode. The vast majority of events from the QCD background do not feature large transverse momentum imbalance and are therefore rejected by the $\alpha_{\mathrm{T}}>0.55$ requirement. The EWK backgrounds consist of $\mathrm{W}$ and $\mathrm{Z}$ boson production, with genuine missing energy due to decay neutrinos. The requirement of at least one b jet greatly reduces the EWK and QCD backgrounds. The dominant background for the analysis arises from $t \bar{t}$ production, in which $\mathrm{b}$ jets and genuine missing energy due to neutrinos can arise from the top quark decay chains.

A procedure based on control data samples, described in section 3.1, is employed to estimate all backgrounds simultaneously. In this method, the fraction of all events with $\alpha_{\mathrm{T}}>0.55$, denoted $\mathrm{F}\left(\alpha_{\mathrm{T}}>0.55\right)$, is measured in a lower- $H_{\mathrm{T}}$ control region and applied in the signal region.

The $\mathrm{Z} \rightarrow \nu \bar{\nu}$ and $t \bar{t}$ background yields are cross-checked separately, as discussed in section 3.2. The $t \bar{t}$ cross-check uses muons to emulate the hadronic decays of taus. The cross-check of $\mathrm{Z} \rightarrow \nu \bar{\nu}$ utilizes $\mathrm{Z} \rightarrow \mu^{+} \mu^{-}$events for which $\alpha_{\mathrm{T}}$ is determined after excluding the muons.

\subsection{Background prediction using $\alpha_{\mathrm{T}}$ vs $H_{\mathrm{T}}$ extrapolation}

In SM simulation studies [10], $\mathrm{F}\left(\alpha_{\mathrm{T}}>0.55\right)$ has no $H_{\mathrm{T}}$ dependence in events with large genuine missing transverse energy, i.e., the $t \bar{t}$ and EWK backgrounds. In the QCD background, however, $\mathrm{F}\left(\alpha_{\mathrm{T}}>0.55\right)$ is expected to be a decreasing function of $H_{\mathrm{T}}$ because of the $H_{\mathrm{T}}$ dependence of the factors contributing to apparent $H_{\mathrm{T}}$, such as jet energy resolution and jet $E_{\mathrm{T}}$ threshold effects.

In data control samples, $\mathrm{F}\left(\alpha_{\mathrm{T}}>0.55\right)$ is consistent with having no $H_{\mathrm{T}}$ dependence, which indicates that the $t \bar{t}$ and EWK backgrounds dominate. The larger anti-tagged data sample is also consistent with having no $H_{\mathrm{T}}$ dependence. Because a tight b-tag requirement further suppresses the QCD background, the tight tagged data sample is expected to have a negligible QCD contribution and therefore $\mathrm{F}\left(\alpha_{\mathrm{T}}>0.55\right)$ independent of $H_{\mathrm{T}}$.

The total background is estimated by measuring $\mathrm{F}\left(\alpha_{\mathrm{T}}>0.55\right)=1.48_{-1.48}^{+1.93} \times 10^{-5}$ in a control region with $250<H_{\mathrm{T}}<350 \mathrm{GeV}$ and multiplying this fraction by the number of events in the signal region before the $\alpha_{\mathrm{T}}>0.55$ requirement. In data, this procedure yields a prediction of $0.33{ }_{-0.33}^{+0.43}$ (stat.) \pm 0.13 (syst.) events. The statistical uncertainty is dominated by the presence of one event with $\alpha_{\mathrm{T}}>0.55$ in the control sample. The systematic uncertainty on the prediction is given by the difference in $\mathrm{F}\left(\alpha_{\mathrm{T}}>0.55\right)$ measured in the tight and loose tagged control samples. Table 1 lists this background prediction, the observation in data, and the expected contribution of SUSY signal for points LM0, LM1, and LMB. 


\begin{tabular}{|l|ccccc|}
\hline N-jets & Background Prediction & Data & LM0 & LM1 & LMB \\
\hline$\geq 2$ & $0.33_{-0.33}^{+0.43}$ (stat.) \pm 0.13 (syst.) & 1 & 14 & 2 & 5 \\
\hline
\end{tabular}

Table 1. Predicted and observed numbers of events for $35 \mathrm{pb}^{-1}$. The prediction comes from the $\alpha_{\mathrm{T}}$ vs $H_{\mathrm{T}}$ extrapolation described in section 3.1 .

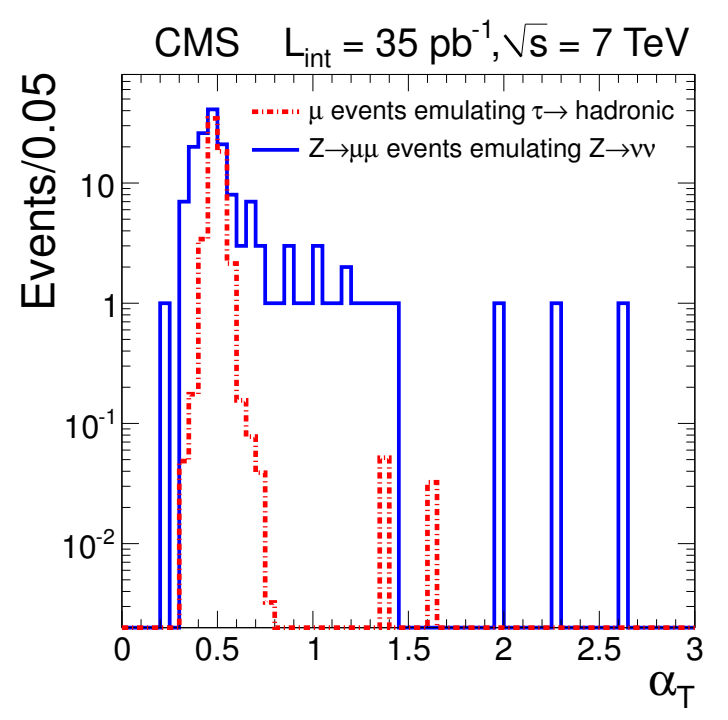

Figure 1. The $\alpha_{\mathrm{T}}$ distributions for $\mathrm{Z} \rightarrow \mu^{+} \mu^{-}$emulation of $\mathrm{Z} \rightarrow \nu \bar{\nu}$ (solid blue) and muon emulation of hadronic tau decays (dashed red).

\subsection{Cross-checks of $\mathrm{Z} \rightarrow \nu \bar{\nu}$ and $t \bar{t}$ background contributions}

While the above background estimate is the one used in this search, we perform auxiliary measurements to cross-check the $\mathrm{Z} \rightarrow \nu \bar{\nu}$ and $t \bar{t}$ background components, which together are expected to comprise the majority of the background. As would be crucial in case of an observed excess, these cross-checks provide an overestimate of the $\mathrm{Z} \rightarrow \nu \bar{\nu}$ and $t \bar{t}$ background components.

For $\mathrm{Z} \rightarrow \nu \bar{\nu}$, a sample of $\mathrm{Z} \rightarrow \mu^{+} \mu^{-}$events is selected with two or more jets but no $\alpha_{\mathrm{T}}$, $H_{\mathrm{T}}$, or b-tagging requirements. The solid blue line in figure 1 shows the $\alpha_{\mathrm{T}}$ distribution for the resulting events. The fraction of these events containing a b-tagged jet is measured. Then, a sample is selected with no b-tag requirement, jet $E_{\mathrm{T}}=75 \mathrm{GeV}$ thresholds on the two highest- $E_{\mathrm{T}}$ jets, $H_{\mathrm{T}}>275 \mathrm{GeV}$, and $\alpha_{\mathrm{T}}>0.52$. The number of events in this sample is scaled by the measured b-tag fraction in the other sample, corrected for the muon identification efficiency and acceptance, and multiplied by the ratio of branching fractions $\frac{\mathrm{BR}(\mathrm{Z} \rightarrow \nu \bar{\nu})}{\operatorname{BR}\left(\mathrm{Z} \rightarrow \mu^{+} \mu^{-}\right)} \approx 6$. This procedure gives an overestimate of the number of $\mathrm{Z} \rightarrow \nu \bar{\nu}$ events in the signal region owing to less stringent requirements than in the final selection, and yields $0.48 \pm 0.39$ events.

Simulation studies indicate that most of the $t \bar{t}$ background comes from events with hadronic tau decays. To estimate the hadronic tau decay yield, $\mathrm{F}\left(\alpha_{\mathrm{T}}>0.55\right)$ is first 


\begin{tabular}{|l|cc|cc|cc|}
\hline & \multicolumn{2}{|c|}{ LM0 } & \multicolumn{2}{c|}{ LM1 } & \multicolumn{2}{c|}{ LMB } \\
Requirement & & & & & & \\
\hline Pre-selection & $98 \%$ & $98 \%$ & $98 \%$ & $98 \%$ & $98 \%$ & $98 \%$ \\
Lepton/Photon Veto & $57 \%$ & $56 \%$ & $55 \%$ & $54 \%$ & $61 \%$ & $60 \%$ \\
Jet Requirements & $51 \%$ & $28 \%$ & $63 \%$ & $34 \%$ & $54 \%$ & $33 \%$ \\
$H_{\mathrm{T}}>350 \mathrm{GeV}$ & $90 \%$ & $25 \%$ & $94 \%$ & $32 \%$ & $97 \%$ & $32 \%$ \\
Trigger & $99 \%$ & $25 \%$ & $99 \%$ & $32 \%$ & $99 \%$ & $31 \%$ \\
Apparent $H_{\mathrm{T}}$ Veto & $68 \%$ & $17 \%$ & $81 \%$ & $26 \%$ & $65 \%$ & $20 \%$ \\
Tight b-tag & $31 \%$ & $5.3 \%$ & $12 \%$ & $3.0 \%$ & $54 \%$ & $11 \%$ \\
$\alpha_{\mathrm{T}}>0.55$ & $14 \%$ & $0.7 \%$ & $29 \%$ & $0.9 \%$ & $12 \%$ & $1.3 \%$ \\
\hline
\end{tabular}

Table 2. Cumulative and individual efficiencies for the selection in three SUSY benchmark points. For each point, the left and right columns represent the individual and cumulative efficiencies, respectively. Different benchmarks have different b-tag efficiencies due to different average numbers of $\mathrm{b}$ quarks per event. The fraction of events containing at least one $\mathrm{b}$ quark before the $\mathrm{b}$-tag selection is $66 \%$ in LM0, $18 \%$ in LM1, and $91 \%$ in LMB.

measured in a sample with $E_{\mathrm{T}}=80 \mathrm{GeV}$ thresholds on the two leading jets, $H_{\mathrm{T}}>280 \mathrm{GeV}$, at least one medium b-tagged jet (TCHP $>1.91$ ), and one or two muons. These selection requirements are chosen to be less strict than the signal selection in order to increase the number of events in this sample. The muons are used to emulate the hadronic decays of taus. To do so, for each muon the presence of a tau jet is emulated with an $E_{\mathrm{T}}$ value set to a fraction of the muon $p_{\mathrm{T}}$, using a distribution taken from simulation. The dashed red line in figure 1 displays the resulting $\alpha_{\mathrm{T}}$ distribution. The measured value of $\mathrm{F}\left(\alpha_{\mathrm{T}}>0.55\right)$ in this sample is multiplied by the number of emulated events in the signal region before the $\alpha_{\mathrm{T}}$ requirement. This value is corrected for the muon selection efficiency, acceptance and the hadronic tau decay branching ratio to obtain the hadronic tau decay yield. The predicted hadronic tau decay yield is increased by $38 \%$, as determined in simulation, in order to account for the entire $t \bar{t}$ background. The procedure yields a $25 \%$ overestimate of the total $t \bar{t}$ background in simulation. In data, $1.4 \pm 0.5$ events are predicted.

\section{Signal selection efficiency}

To interpret the results of this search in terms of a given signal model, the selection efficiency for that model must be determined. Table 2 lists the cumulative and individual efficiencies for the event selection in the three SUSY benchmark models LM0, LM1, and LMB, from which events are generated at leading order (LO) via PYTHIA 6.4, tune Z2 [17] using parton distribution functions provided by CTEQ6.6 [18]. Without b tagging, the cumulative efficiencies for LM0 and LM1 are about $85 \%$ of those in ref. [10], because of a more stringent lepton and photon veto. Table 3 lists the relative systematic uncertainties on the signal yield, which are dominated by the uncertainty on the b-tagging efficiency, described below. The other uncertainties and the methods used to obtain them are similar to ref. [10]. 


\begin{tabular}{|lr|}
\hline Source & Uncertainty $(\%)$ \\
\hline Luminosity & 4 \\
JES & 3.5 \\
Jet Energy Resolution & 1 \\
Trigger Efficiency & 1 \\
Apparent $H_{\mathrm{T}}$ Veto & 4 \\
Lepton/Photon Veto & 4 \\
b-tag Efficiency (LMB) & 20 \\
\hline Total & 22 \\
\hline
\end{tabular}

Table 3. Relative systematic uncertainties on the signal yield.

The b-tagging efficiency is measured from inclusive dijet events in which one jet has an associated muon and another "away" jet has a TCHP value of at least 1.0. The relative fraction of jets from $b$ quarks in a data sample is determined by a fit to the distribution of transverse momentum of muons relative to their associated jet axis, $p_{\mathrm{T}}^{\mathrm{rel}}[9,19]$, which is larger for jets from $b$ quarks than from other flavours. This fit is to a linear combination of simulation-derived $p_{\mathrm{T}}^{\text {rel }}$ expected distributions from different flavours. The fitted $\mathrm{b}$ fractions for jets passing and failing the analysis b-tagging requirement are used in the b-tagging efficiency calculation. This efficiency is measured separately for jets with $|\eta|>1.4$ and $|\eta| \leq$ 1.4, in four ranges of jet $E_{\mathrm{T}}$. The ratio between the b-tagging efficiency measured in data and in simulation is taken as the efficiency scale factor for a particular range in $E_{\mathrm{T}}$ and $|\eta|$.

Systematic uncertainties on the scale factors arise from potential biases in the $p_{\mathrm{T}}^{\mathrm{rel}}$ fitting procedure. These uncertainties are measured by varying the muon-to-jet matching and muon $p_{\mathrm{T}}$ thresholds, fraction of gluon splitting to $b \bar{b}$, jet energy scale and resolution, jet angular resolution, and b-tagging requirement on the away jet. The effect of measuring the scale factors using only semi-leptonic b decays is also accounted for. The scale factors are used to correct the expected event yield at each signal point for differences between the efficiencies in data and simulation. For example, for LMB the application of the scale factors translates into a change in the yield by a factor $0.87 \pm 0.18$. The systematic and statistical uncertainties give a total relative uncertainty of $20 \%$ in LMB, with a similar uncertainty of $23 \%$ in LM1.

\section{Results}

The observation of one data event in the signal region is consistent with background expectations. Combining the expected signal and background prediction from section 3.1 and using frequentist statistical methods in the manner of ref. [20] with the Profile Likelihood ratio [21] to handle nuisance parameters, we derive 95\% confidence level (CL) cross-section upper limits $\left(\sigma_{95}^{\text {obs }}\right)$ of $18.9,15.4$, and $10.2 \mathrm{pb}$ for LM0, LM1, and LMB, respectively. The effect of possibly overestimating the background due to signal contamination in the control regions increases the $\sigma_{95}^{\text {obs }}$ value to $22.1 \mathrm{pb}$ for LM0, $16.7 \mathrm{pb}$ for LM1, but is negligible 


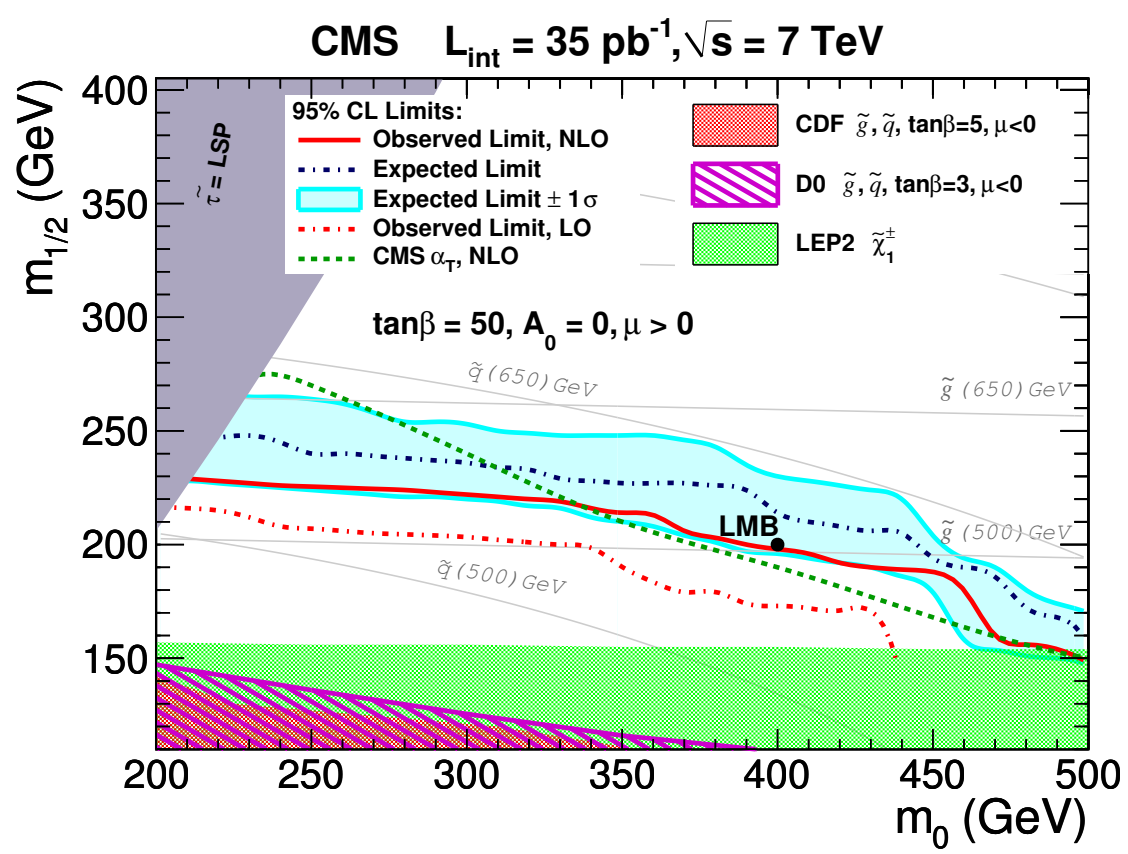

Figure 2. Exclusion regions in the $\left(m_{0}, m_{1 / 2}\right)$ plane for one set of CMSSM parameters, for this analysis (solid red), and the non-b tagged version [10] (dashed green).

for LMB. To quantify the sensitivity with reduced dependence on the amount of b-quark production, a 95\% CL upper limit on the cross section times branching ratio to at least one b quark of $4.0 \mathrm{pb}$ is determined in LM1.

The resulting excluded region in the $\left(m_{0}, m_{1 / 2}\right)$ plane for a reference model with CMSSM parameters $A_{0}=0 \mathrm{GeV}, \tan \beta=50$, and $\mu>0$ is shown in figure 2. The expected and observed exclusion regions are calculated using next-to-leading-order (NLO) cross sections, obtained with the program Prospino [22]. The excluded region is extended with respect to that of ref. [10] without b tagging, also shown, for scenarios with increased b production, such as those with $m_{0}$ above $350 \mathrm{GeV}$.

\section{Summary}

A search for events with multiple jets, at least one of which is b tagged, and significant transverse momentum imbalance has been presented. One event is observed, which is consistent with background expectations. The dominant background comes from $t \bar{t}$ production. The results of the search are characterized as an exclusion region in CMSSM parameter space and 95\% CL upper limits on representative scenarios with expected cross section of approximately $15 \mathrm{pb}$. The sensitivity of this search surpasses that of the Tevatron experiments $[23,24]$ and is comparable to a recent fully hadronic, b-tagged search from ATLAS [25]. 


\section{Acknowledgments}

We wish to congratulate our colleagues in the CERN accelerator departments for the excellent performance of the LHC machine. We thank the technical and administrative staff at CERN and other CMS institutes, and acknowledge support from: FMSR (Austria); FNRS and FWO (Belgium); CNPq, CAPES, FAPERJ, and FAPESP (Brazil); MES (Bulgaria); CERN; CAS, MoST, and NSFC (China); COLCIENCIAS (Colombia); MSES (Croatia); RPF (Cyprus); Academy of Sciences and NICPB (Estonia); Academy of Finland, MEC, and HIP (Finland); CEA and CNRS/IN2P3 (France); BMBF, DFG, and HGF (Germany); GSRT (Greece); OTKA and NKTH (Hungary); DAE and DST (India); IPM (Iran); SFI (Ireland); INFN (Italy); NRF and WCU (Korea); LAS (Lithuania); CINVESTAV, CONACYT, SEP, and UASLP-FAI (Mexico); MSI (New Zealand); PAEC (Pakistan); SCSR (Poland); FCT (Portugal); JINR (Armenia, Belarus, Georgia, Ukraine, Uzbekistan); MST, MAE and RFBR (Russia); MSTD (Serbia); MICINN and CPAN (Spain); Swiss Funding Agencies (Switzerland); NSC (Taipei); TUBITAK and TAEK (Turkey); STFC (United Kingdom); DOE and NSF (USA).

Open Access. This article is distributed under the terms of the Creative Commons Attribution Noncommercial License which permits any noncommercial use, distribution, and reproduction in any medium, provided the original author(s) and source are credited.

\section{References}

[1] J. Wess and B. Zumino, Supergauge transformations in four-dimensions, Nucl. Phys. B 70 (1974) 39 [SPIRES].

[2] H.P. Nilles, Supersymmetry, supergravity and particle physics, Phys. Reports 110 (1984) 1.

[3] H.E. Haber and G.L. Kane, The search for supersymmetry: probing physics beyond the standard model, Phys. Reports 117 (1987) 75.

[4] R. Barbieri, S. Ferrara and C.A. Savoy, Gauge models with spontaneously broken local supersymmetry, Phys. Lett. B 119 (1982) 343 [SPIRES].

[5] S. Dawson, E. Eichten and C. Quigg, Search for supersymmetric particles in hadron-hadron collisions, Phys. Rev. D 31 (1985) 1581 [SPIRES].

[6] E. Witten, Dynamical breaking of supersymmetry, Nucl. Phys. B 188 (1981) 513 [SPIRES].

[7] S. Dimopoulos and H. Georgi, Softly broken supersymmetry and SU(5), Nucl. Phys. B 193 (1981) 150 [SPIRES].

[8] G. Jungman, M. Kamionkowski and K. Griest, Supersymmetric dark matter, Phys. Rept. 267 (1996) 195 [hep-ph/9506380] [SPIRES].

[9] CMS collaboration, Commissioning of b-jet identification with pp collisions at $\sqrt{s}=7 \mathrm{TeV}$, CMS Physics Analysis Summary BTV-10-001 (2010).

[10] CMS collaboration, V. Khachatryan et al., Search for supersymmetry in pp collisions at $7 \mathrm{TeV}$ in events with jets and missing transverse energy, Phys. Lett. B 698 (2011) 196 [arXiv:1101.1628] [SPIRES]. 
[11] L. Randall and D. Tucker-Smith, Dijet searches for supersymmetry at the LHC, Phys. Rev. Lett. 101 (2008) 221803 [arXiv: 0806.1049] [SPIRES].

[12] A.H. Chamseddine, R.L. Arnowitt and P. Nath, Locally supersymmetric grand unification, Phys. Rev. Lett. 49 (1982) 970 [SPIRES].

[13] G.L. Kane, C.F. Kolda, L. Roszkowski and J.D. Wells, Study of constrained minimal supersymmetry, Phys. Rev. D 49 (1994) 6173 [hep-ph/9312272] [SPIRES].

[14] CMS collaboration, The CMS experiment at the CERN LHC, JINST 03 (2008) S08004.

[15] CMS collaboration, Tracking and primary vertex results in first $7 \mathrm{TeV}$ collisions, CMS Physics Analysis Summary TRK-10-005 (2010).

[16] M. Cacciari, G.P. Salam and G. Soyez, The anti- $k_{t}$ jet clustering algorithm, JHEP 04 (2008) 063 [arXiv: 0802.1189] [SPIRES].

[17] T. Sjöstrand, S. Mrenna and P.Z. Skands, PYTHIA 6.4 physics and manual, JHEP 05 (2006) 026 [hep-ph/0603175] [SPIRES].

[18] P.M. Nadolsky et al., Implications of CTEQ global analysis for collider observables, Phys. Rev. D 78 (2008) 013004 [arXiv: 0802.0007] [SPIRES].

[19] CMS collaboration, V. Khachatryan et al., Inclusive b-hadron production cross section with muons in pp collisions at $\sqrt{s}=7 \mathrm{TeV}$, JHEP 03 (2011) 090 [arXiv:1101.3512] [SPIRES].

[20] G.J. Feldman and R.D. Cousins, A unified approach to the classical statistical analysis of small signals, Phys. Rev. D 57 (1998) 3873 [physics/9711021].

[21] T.A. Severini, Likelihood methods in statistics, Oxford University Press, Oxford U.K. (2000).

[22] W. Beenakker, R. Hopker, M. Spira and P.M. Zerwas, Squark and gluino production at hadron colliders, Nucl. Phys. B 492 (1997) 51 [hep-ph/9610490] [SPIRES].

[23] CDF collaboration, T. Aaltonen et al., Inclusive search for squark and gluino production in $p \bar{p}$ collisions at $\sqrt{s}=1.96 \mathrm{TeV}$, Phys. Rev. Lett. 102 (2009) 121801 [arXiv:0811.2512] [SPIRES].

[24] D0 collaboration, V.M. Abazov et al., Search for squarks and gluinos in events with jets and missing transverse energy using $2.1 \mathrm{fb}^{-1}$ of p $\bar{p}$ collision data at $\sqrt{s}=1.96 \mathrm{TeV}$, Phys. Lett. B 660 (2008) 449 [arXiv:0712.3805] [SPIRES].

[25] ATLAS collaboration, G. Aad et al., Search for supersymmetry in pp collisions at $\sqrt{s}=7 \mathrm{TeV}$ in final states with missing transverse momentum and b-jets, Phys. Lett. B 701 (2011) 398 [arXiv:1103.4344] [SPIRES]. 


\section{The CMS collaboration}

\section{Yerevan Physics Institute, Yerevan, Armenia}

S. Chatrchyan, V. Khachatryan, A.M. Sirunyan, A. Tumasyan

Institut für Hochenergiephysik der OeAW, Wien, Austria

W. Adam, T. Bergauer, M. Dragicevic, J. Erö, C. Fabjan, M. Friedl, R. Frühwirth, V.M. Ghete, J. Hammer ${ }^{1}$, S. Hänsel, M. Hoch, N. Hörmann, J. Hrubec, M. Jeitler, W. Kiesenhofer, M. Krammer, D. Liko, I. Mikulec, M. Pernicka, H. Rohringer, R. Schöfbeck, J. Strauss, A. Taurok, F. Teischinger, P. Wagner, W. Waltenberger, G. Walzel, E. Widl, C.-E. Wulz

National Centre for Particle and High Energy Physics, Minsk, Belarus

V. Mossolov, N. Shumeiko, J. Suarez Gonzalez

Universiteit Antwerpen, Antwerpen, Belgium

S. Bansal, L. Benucci, E.A. De Wolf, X. Janssen, J. Maes, T. Maes, L. Mucibello, S. Ochesanu, B. Roland, R. Rougny, M. Selvaggi, H. Van Haevermaet, P. Van Mechelen, N. Van Remortel

\section{Vrije Universiteit Brussel, Brussel, Belgium}

F. Blekman, S. Blyweert, J. D'Hondt, O. Devroede, R. Gonzalez Suarez, A. Kalogeropoulos, M. Maes, W. Van Doninck, P. Van Mulders, G.P. Van Onsem, I. Villella

Université Libre de Bruxelles, Bruxelles, Belgium

O. Charaf, B. Clerbaux, G. De Lentdecker, V. Dero, A.P.R. Gay, G.H. Hammad, T. Hreus, P.E. Marage, L. Thomas, C. Vander Velde, P. Vanlaer

Ghent University, Ghent, Belgium

V. Adler, A. Cimmino, S. Costantini, M. Grunewald, B. Klein, J. Lellouch, A. Marinov, J. Mccartin, D. Ryckbosch, F. Thyssen, M. Tytgat, L. Vanelderen, P. Verwilligen, S. Walsh,

N. Zaganidis

Université Catholique de Louvain, Louvain-la-Neuve, Belgium

S. Basegmez, G. Bruno, J. Caudron, L. Ceard, E. Cortina Gil, J. De Favereau De Jeneret, C. Delaere ${ }^{1}$, D. Favart, A. Giammanco, G. Grégoire, J. Hollar, V. Lemaitre, J. Liao, O. Militaru, C. Nuttens, S. Ovyn, D. Pagano, A. Pin, K. Piotrzkowski, N. Schul

Université de Mons, Mons, Belgium

N. Beliy, T. Caebergs, E. Daubie

Centro Brasileiro de Pesquisas Fisicas, Rio de Janeiro, Brazil

G.A. Alves, D. De Jesus Damiao, M.E. Pol, M.H.G. Souza

Universidade do Estado do Rio de Janeiro, Rio de Janeiro, Brazil

W. Carvalho, E.M. Da Costa, C. De Oliveira Martins, S. Fonseca De Souza, L. Mundim, H. Nogima, V. Oguri, W.L. Prado Da Silva, A. Santoro, S.M. Silva Do Amaral, A. Sznajder Instituto de Fisica Teorica, Universidade Estadual Paulista, Sao Paulo, Brazil C.A. Bernardes ${ }^{2}$, F.A. Dias, T.R. Fernandez Perez Tomei, E. M. Gregores², C. Lagana, F. Marinho, P.G. Mercadante ${ }^{2}$, S.F. Novaes, Sandra S. Padula 
Institute for Nuclear Research and Nuclear Energy, Sofia, Bulgaria

N. Darmenov ${ }^{1}$, V. Genchev ${ }^{1}$, P. Iaydjiev ${ }^{1}$, S. Piperov, M. Rodozov, S. Stoykova,

G. Sultanov, V. Tcholakov, R. Trayanov

University of Sofia, Sofia, Bulgaria

A. Dimitrov, R. Hadjiiska, A. Karadzhinova, V. Kozhuharov, L. Litov, M. Mateev,

B. Pavlov, P. Petkov

Institute of High Energy Physics, Beijing, China

J.G. Bian, G.M. Chen, H.S. Chen, C.H. Jiang, D. Liang, S. Liang, X. Meng, J. Tao, J. Wang, J. Wang, X. Wang, Z. Wang, H. Xiao, M. Xu, J. Zang, Z. Zhang

State Key Lab. of Nucl. Phys. and Tech., Peking University, Beijing, China

Y. Ban, S. Guo, Y. Guo, W. Li, Y. Mao, S.J. Qian, H. Teng, B. Zhu, W. Zou

Universidad de Los Andes, Bogota, Colombia

A. Cabrera, B. Gomez Moreno, A.A. Ocampo Rios, A.F. Osorio Oliveros, J.C. Sanabria

Technical University of Split, Split, Croatia

N. Godinovic, D. Lelas, K. Lelas, R. Plestina ${ }^{3}$, D. Polic, I. Puljak

University of Split, Split, Croatia

Z. Antunovic, M. Dzelalija

Institute Rudjer Boskovic, Zagreb, Croatia

V. Brigljevic, S. Duric, K. Kadija, S. Morovic

University of Cyprus, Nicosia, Cyprus

A. Attikis, M. Galanti, J. Mousa, C. Nicolaou, F. Ptochos, P.A. Razis

Charles University, Prague, Czech Republic

M. Finger, M. Finger Jr.

Academy of Scientific Research and Technology of the Arab Republic of Egypt, Egyptian Network of High Energy Physics, Cairo, Egypt

Y. Assran ${ }^{4}$, S. Khalil ${ }^{5}$, M.A. Mahmoud ${ }^{6}$

National Institute of Chemical Physics and Biophysics, Tallinn, Estonia

A. Hektor, M. Kadastik, M. Müntel, M. Raidal, L. Rebane

Department of Physics, University of Helsinki, Helsinki, Finland

V. Azzolini, P. Eerola, G. Fedi

Helsinki Institute of Physics, Helsinki, Finland

S. Czellar, J. Härkönen, A. Heikkinen, V. Karimäki, R. Kinnunen, M.J. Kortelainen, T. Lampén, K. Lassila-Perini, S. Lehti, T. Lindén, P. Luukka, T. Mäenpää, E. Tuominen, J. Tuominiemi, E. Tuovinen, D. Ungaro, L. Wendland

Lappeenranta University of Technology, Lappeenranta, Finland

K. Banzuzi, A. Karjalainen, A. Korpela, T. Tuuva

Laboratoire d'Annecy-le-Vieux de Physique des Particules, IN2P3-CNRS, Annecy-le-Vieux, France

D. Sillou 
DSM/IRFU, CEA/Saclay, Gif-sur-Yvette, France

M. Besancon, S. Choudhury, M. Dejardin, D. Denegri, B. Fabbro, J.L. Faure, F. Ferri,

S. Ganjour, F.X. Gentit, A. Givernaud, P. Gras, G. Hamel de Monchenault, P. Jarry,

E. Locci, J. Malcles, M. Marionneau, L. Millischer, J. Rander, A. Rosowsky, I. Shreyber, M. Titov, P. Verrecchia

Laboratoire Leprince-Ringuet, Ecole Polytechnique, IN2P3-CNRS, Palaiseau, France

S. Baffioni, F. Beaudette, L. Benhabib, L. Bianchini, M. Bluj ${ }^{7}$, C. Broutin, P. Busson, C. Charlot, T. Dahms, L. Dobrzynski, S. Elgammal, R. Granier de Cassagnac, M. Haguenauer, P. Miné, C. Mironov, C. Ochando, P. Paganini, D. Sabes, R. Salerno, Y. Sirois, C. Thiebaux, B. Wyslouch ${ }^{8}$, A. Zabi

Institut Pluridisciplinaire Hubert Curien, Université de Strasbourg, Université de Haute Alsace Mulhouse, CNRS/IN2P3, Strasbourg, France

J.-L. Agram ${ }^{9}$, J. Andrea, D. Bloch, D. Bodin, J.-M. Brom, M. Cardaci, E.C. Chabert, C. Collard, E. Conte ${ }^{9}$, F. Drouhin ${ }^{9}$, C. Ferro, J.-C. Fontaine ${ }^{9}$, D. Gelé, U. Goerlach, S. Greder, P. Juillot, M. Karim ${ }^{9}$, A.-C. Le Bihan, Y. Mikami, P. Van Hove

Centre de Calcul de l'Institut National de Physique Nucleaire et de Physique des Particules (IN2P3), Villeurbanne, France

F. Fassi, D. Mercier

Université de Lyon, Université Claude Bernard Lyon 1, CNRS-IN2P3, Institut de Physique Nucléaire de Lyon, Villeurbanne, France

C. Baty, S. Beauceron, N. Beaupere, M. Bedjidian, O. Bondu, G. Boudoul, D. Boumediene,

H. Brun, J. Chasserat, R. Chierici, D. Contardo, P. Depasse, H. El Mamouni, J. Fay,

S. Gascon, B. Ille, T. Kurca, T. Le Grand, M. Lethuillier, L. Mirabito, S. Perries, V. Sordini,

S. Tosi, Y. Tschudi, P. Verdier

Institute of High Energy Physics and Informatization, Tbilisi State University, Tbilisi, Georgia

D. Lomidze

RWTH Aachen University, I. Physikalisches Institut, Aachen, Germany

G. Anagnostou, S. Beranek, M. Edelhoff, L. Feld, N. Heracleous, O. Hindrichs, R. Jussen, K. Klein, J. Merz, N. Mohr, A. Ostapchuk, A. Perieanu, F. Raupach, J. Sammet, S. Schael, D. Sprenger, H. Weber, M. Weber, B. Wittmer

RWTH Aachen University, III. Physikalisches Institut A, Aachen, Germany

M. Ata, E. Dietz-Laursonn, M. Erdmann, T. Hebbeker, A. Hinzmann, K. Hoepfner, T. Klimkovich, D. Klingebiel, P. Kreuzer, D. Lanske ${ }^{\dagger}$, C. Magass, M. Merschmeyer, A. Meyer, P. Papacz, H. Pieta, H. Reithler, S.A. Schmitz, L. Sonnenschein, J. Steggemann, D. Teyssier

RWTH Aachen University, III. Physikalisches Institut B, Aachen, Germany

M. Bontenackels, M. Davids, M. Duda, G. Flügge, H. Geenen, M. Giffels, W. Haj Ahmad, D. Heydhausen, F. Hoehle, B. Kargoll, T. Kress, Y. Kuessel, A. Linn, A. Nowack, L. Perchalla, O. Pooth, J. Rennefeld, P. Sauerland, A. Stahl, M. Thomas, D. Tornier, M.H. Zoeller 


\section{Deutsches Elektronen-Synchrotron, Hamburg, Germany}

M. Aldaya Martin, W. Behrenhoff, U. Behrens, M. Bergholz ${ }^{10}$, A. Bethani, K. Borras,

A. Cakir, A. Campbell, E. Castro, D. Dammann, G. Eckerlin, D. Eckstein, A. Flossdorf, G. Flucke, A. Geiser, J. Hauk, H. Jung ${ }^{1}$, M. Kasemann, I. Katkov ${ }^{11}$, P. Katsas, C. Kleinwort, H. Kluge, A. Knutsson, M. Krämer, D. Krücker, E. Kuznetsova, W. Lange, W. Lohmann ${ }^{10}$, R. Mankel, M. Marienfeld, I.-A. Melzer-Pellmann, A.B. Meyer, J. Mnich, A. Mussgiller, J. Olzem, A. Petrukhin, D. Pitzl, A. Raspereza, A. Raval, M. Rosin, R. Schmidt ${ }^{10}$, T. Schoerner-Sadenius, N. Sen, A. Spiridonov, M. Stein, J. Tomaszewska, R. Walsh, C. Wissing

\section{University of Hamburg, Hamburg, Germany}

C. Autermann, V. Blobel, S. Bobrovskyi, J. Draeger, H. Enderle, U. Gebbert, M. Görner, K. Kaschube, G. Kaussen, H. Kirschenmann, R. Klanner, J. Lange, B. Mura, S. NaumannEmme, F. Nowak, N. Pietsch, C. Sander, H. Schettler, P. Schleper, E. Schlieckau, M. Schröder, T. Schum, J. Schwandt, H. Stadie, G. Steinbrück, J. Thomsen

Institut für Experimentelle Kernphysik, Karlsruhe, Germany

C. Barth, J. Bauer, J. Berger, V. Buege, T. Chwalek, W. De Boer, A. Dierlamm, G. Dirkes, M. Feindt, J. Gruschke, C. Hackstein, F. Hartmann, M. Heinrich, H. Held, K.H. Hoffmann, S. Honc, J.R. Komaragiri, T. Kuhr, D. Martschei, S. Mueller, Th. Müller, M. Niegel, O. Oberst, A. Oehler, J. Ott, T. Peiffer, G. Quast, K. Rabbertz, F. Ratnikov, N. Ratnikova, M. Renz, C. Saout, A. Scheurer, P. Schieferdecker, F.-P. Schilling, G. Schott, H.J. Simonis, F.M. Stober, D. Troendle, J. Wagner-Kuhr, T. Weiler, M. Zeise, V. Zhukov ${ }^{11}$, E.B. Ziebarth

Institute of Nuclear Physics "Demokritos", Aghia Paraskevi, Greece

G. Daskalakis, T. Geralis, S. Kesisoglou, A. Kyriakis, D. Loukas, I. Manolakos, A. Markou, C. Markou, C. Mavrommatis, E. Ntomari, E. Petrakou

University of Athens, Athens, Greece

L. Gouskos, T.J. Mertzimekis, A. Panagiotou, E. Stiliaris

University of Ioánnina, Ioánnina, Greece

I. Evangelou, C. Foudas, P. Kokkas, N. Manthos, I. Papadopoulos, V. Patras, F.A. Triantis

KFKI Research Institute for Particle and Nuclear Physics, Budapest, Hungary A. Aranyi, G. Bencze, L. Boldizsar, C. Hajdu ${ }^{1}$, P. Hidas, D. Horvath ${ }^{12}$, A. Kapusi, K. Krajczar ${ }^{13}$, F. Sikler ${ }^{1}$, G.I. Veres ${ }^{13}$, G. Vesztergombi ${ }^{13}$

Institute of Nuclear Research ATOMKI, Debrecen, Hungary

N. Beni, J. Molnar, J. Palinkas, Z. Szillasi, V. Veszpremi

\section{University of Debrecen, Debrecen, Hungary}

P. Raics, Z.L. Trocsanyi, B. Ujvari

\section{Panjab University, Chandigarh, India}

S.B. Beri, V. Bhatnagar, N. Dhingra, R. Gupta, M. Jindal, M. Kaur, J.M. Kohli, M.Z. Mehta, N. Nishu, L.K. Saini, A. Sharma, A.P. Singh, J. Singh, S.P. Singh 
University of Delhi, Delhi, India

S. Ahuja, B.C. Choudhary, P. Gupta, S. Jain, S. Jain, A. Kumar, A. Kumar, M. Naimuddin, K. Ranjan, R.K. Shivpuri

Saha Institute of Nuclear Physics, Kolkata, India

S. Bhattacharya, S. Dutta, B. Gomber, R. Khurana, S. Sarkar

Bhabha Atomic Research Centre, Mumbai, India

R.K. Choudhury, D. Dutta, S. Kailas, V. Kumar, P. Mehta, A.K. Mohanty ${ }^{1}$, L.M. Pant, P. Shukla

Tata Institute of Fundamental Research - EHEP, Mumbai, India

T. Aziz, M. Guchait ${ }^{14}$, A. Gurtu, M. Maity ${ }^{15}$, D. Majumder, G. Majumder, K. Mazumdar, G.B. Mohanty, A. Saha, K. Sudhakar, N. Wickramage

Tata Institute of Fundamental Research - HECR, Mumbai, India

S. Banerjee, S. Dugad, N.K. Mondal

Institute for Research and Fundamental Sciences (IPM), Tehran, Iran

H. Arfaei, H. Bakhshiansohi ${ }^{16}$, S.M. Etesami, A. Fahim ${ }^{16}$, M. Hashemi, A. Jafari ${ }^{16}$, M. Khakzad, A. Mohammadi ${ }^{17}$, M. Mohammadi Najafabadi, S. Paktinat Mehdiabadi, B. Safarzadeh, M. Zeinali ${ }^{18}$

INFN Sezione di Bari ${ }^{a}$, Università di Bari ${ }^{b}$, Politecnico di Bari ${ }^{c}$, Bari, Italy

M. Abbrescia ${ }^{a, b}$, L. Barbone ${ }^{a, b}$, C. Calabria ${ }^{a, b}$, A. Colaleo ${ }^{a}$, D. Creanza ${ }^{a, c}$, N. De Filippis $^{a, c, 1}$, M. De Palma ${ }^{a, b}$, L. Fiore $^{a}$, G. Iaselli ${ }^{a, c}$, L. Lusito $^{a, b}$, G. Maggi $^{a, c}$, M. Maggi $^{a}$, N. Manna ${ }^{a, b}$, B. Marangelli ${ }^{a, b}$, S. My ${ }^{a, c}$, S. Nuzzo ${ }^{a, b}$, N. Pacifico ${ }^{a, b}$, G.A. Pierro ${ }^{a}$, A. Pompili ${ }^{a, b}$, G. Pugliese ${ }^{a, c}$, F. Romano ${ }^{a, c}$, G. Roselli ${ }^{a, b}$, G. Selvaggi ${ }^{a, b}$, L. Silvestris ${ }^{a}$, R. Trentadue ${ }^{a}$, S. Tupputi ${ }^{a, b}$, G. Zito ${ }^{a}$

INFN Sezione di Bologna ${ }^{a}$, Università di Bologna ${ }^{b}$, Bologna, Italy

G. Abbiendi ${ }^{a}$, A.C. Benvenuti ${ }^{a}$, D. Bonacorsi ${ }^{a}$, S. Braibant-Giacomelli $^{a}, b$, L. Brigliadori ${ }^{a}$, P. Capiluppi ${ }^{a, b}$, A. Castro ${ }^{a, b}$, F.R. Cavallo ${ }^{a}$, M. Cuffiani ${ }^{a, b}$, G.M. Dallavalle ${ }^{a}$, F. Fabbri $^{a}$, A. Fanfani ${ }^{a}, b$, D. Fasanella ${ }^{a}$, P. Giacomelli ${ }^{a}$, M. Giunta ${ }^{a}$, C. Grandi ${ }^{a}$, S. Marcellini ${ }^{a}$, G. Masetti ${ }^{b}$, M. Meneghelli ${ }^{a}, b$, A. Montanari ${ }^{a}$, F.L. Navarria ${ }^{a}, b$, F. Odorici ${ }^{a}$, A. Perrotta $^{a}$,

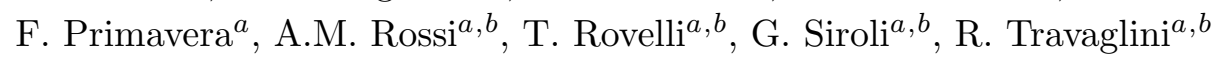

INFN Sezione di Catania ${ }^{a}$, Università di Catania ${ }^{b}$, Catania, Italy

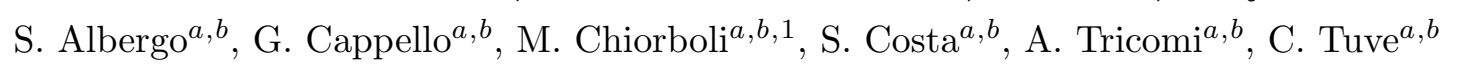

INFN Sezione di Firenze ${ }^{a}$, Università di Firenze ${ }^{b}$, Firenze, Italy

G. Barbagli ${ }^{a}$, V. Ciulli ${ }^{a, b}$, C. Civinini ${ }^{a}$, R. D’Alessandro ${ }^{a, b}$, E. Focardi ${ }^{a}, b$, S. Frosali ${ }^{a, b}$,

E. Gallo ${ }^{a}$, S. Gonzi ${ }^{a, b}$, P. Lenzi ${ }^{a, b}$, M. Meschini ${ }^{a}$, S. Paoletti ${ }^{a}$, G. Sguazzoni ${ }^{a}$,

A. Tropiano ${ }^{a, 1}$

INFN Laboratori Nazionali di Frascati, Frascati, Italy

L. Benussi, S. Bianco, S. Colafranceschi ${ }^{19}$, F. Fabbri, D. Piccolo 
INFN Sezione di Genova, Genova, Italy

P. Fabbricatore, R. Musenich

INFN Sezione di Milano-Bicocca ${ }^{a}$, Università di Milano-Bicocca ${ }^{b}$, Milano, Italy

A. Benaglia ${ }^{a, b}$, F. De Guio ${ }^{a, b, 1}$, L. Di Matteo ${ }^{a, b}$, S. Gennai ${ }^{1}$, A. Ghezzi $^{a, b}$, S. Malvezzi $^{a}$,

A. Martelli ${ }^{a, b}$, A. Massironi ${ }^{a, b}$, D. Menasce ${ }^{a}$, L. Moroni ${ }^{a}$, M. Paganoni ${ }^{a}, b$, D. Pedrini ${ }^{a}$,

S. Ragazzi ${ }^{a, b}$, N. Redaelli ${ }^{a}$, S. Sala ${ }^{a}$, T. Tabarelli de Fatis ${ }^{a, b}$

INFN Sezione di Napoli ${ }^{a}$, Università di Napoli "Federico II" $b$, Napoli, Italy

S. Buontempo ${ }^{a}$, C.A. Carrillo Montoya ${ }^{a, 1}$, N. Cavallo ${ }^{a, 20}$, A. De Cosa ${ }^{a}, b$, F. Fabozzi $^{a, 20}$,

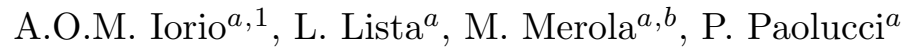

INFN Sezione di Padova ${ }^{a}$, Università di Padova ${ }^{b}$ Università di Trento (Trento) ${ }^{c}$, Padova, Italy

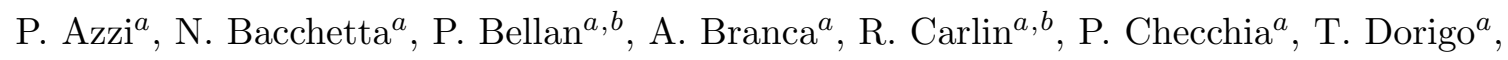

U. Dosselli ${ }^{a}$, F. Gasparini ${ }^{a, b}$, A. Gozzelino, A. Kaminskiy ${ }^{a, b, 11}$, S. Lacaprara ${ }^{a, 21}$,

I. Lazzizzera ${ }^{a, c}$, M. Margoni ${ }^{a, b}$, M. Mazzucato ${ }^{a}$, A.T. Meneguzzo ${ }^{a, b}$, M. Nespolo ${ }^{a, 1}$,

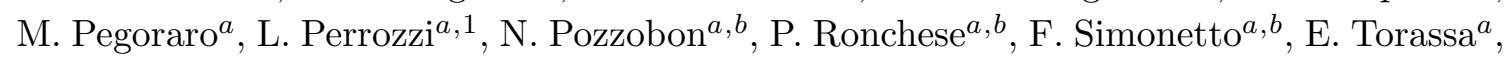

M. Tosi ${ }^{a, b}$, S. Vanini ${ }^{a, b}$, S. Ventura ${ }^{a}$, P. Zotto ${ }^{a, b}$, G. Zumerle ${ }^{a, b}$

INFN Sezione di Pavia ${ }^{a}$, Università di Pavia ${ }^{b}$, Pavia, Italy

P. Baesso ${ }^{a, b}$, U. Berzano ${ }^{a}$, S.P. Ratti ${ }^{a, b}$, C. Riccardi ${ }^{a, b}$, P. Torre ${ }^{a, b}$, P. Vitulo ${ }^{a, b}$, C. Viviani ${ }^{a, b}$

INFN Sezione di Perugia ${ }^{a}$, Università di Perugia ${ }^{b}$, Perugia, Italy

M. Biasini ${ }^{a, b}$, G.M. Bilei ${ }^{a}$, B. Caponeri ${ }^{a, b}$, L. Fanò ${ }^{a, b}$, P. Lariccia ${ }^{a, b}$, A. Lucaroni ${ }^{a, b, 1}$, G. Mantovani ${ }^{a, b}$, M. Menichelli ${ }^{a}$, A. Nappi $^{a, b}$, F. Romeo $^{a, b}$, A. Santocchia $^{a, b}$, S. Taroni $^{a, b, 1}$, M. Valdata ${ }^{a, b}$

INFN Sezione di Pisa ${ }^{a}$, Università di $\mathrm{Pisa}^{b}$, Scuola Normale Superiore di Pisa ${ }^{c}$, Pisa, Italy

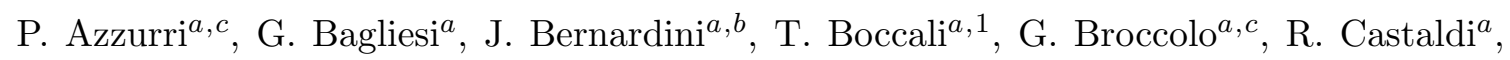

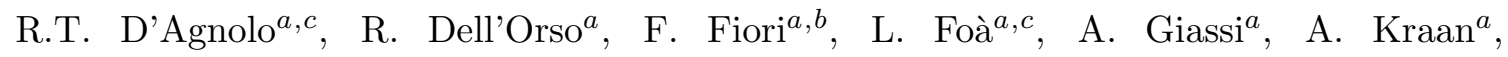

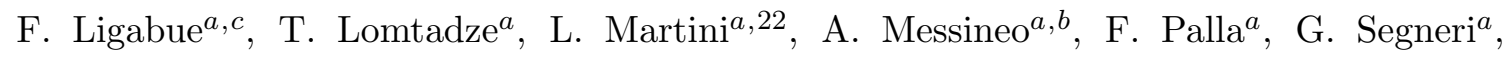

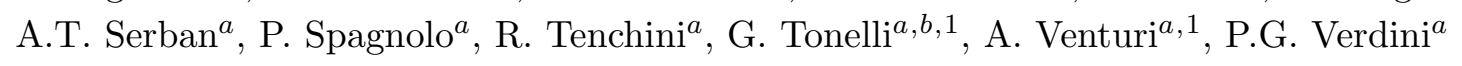

INFN Sezione di Roma ${ }^{a}$, Università di Roma "La Sapienza" $b$, Roma, Italy

L. Barone ${ }^{a, b}$, F. Cavallari ${ }^{a}$, D. Del Re ${ }^{a, b}$, E. Di $\mathrm{Marco}^{a, b}$, M. Diemoz ${ }^{a}$, D. Franci ${ }^{a, b}$,

M. Grassi ${ }^{a, 1}$, E. Longo ${ }^{a, b}$, P. Meridiani, S. Nourbakhsh ${ }^{a}$, G. Organtini ${ }^{a, b}$, F. Pandolfi ${ }^{a, b, 1}$, R. Paramatti ${ }^{a}$, S. Rahatlou ${ }^{a, b}$, C. Rovelli ${ }^{1}$

INFN Sezione di Torino ${ }^{a}$, Università di Torino ${ }^{b}$, Università del Piemonte Orientale (Novara) ${ }^{c}$, Torino, Italy

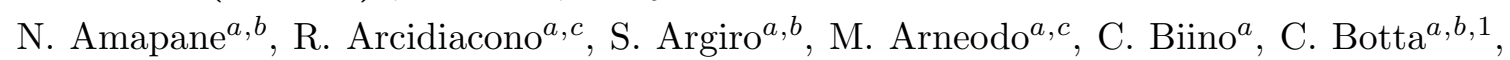

N. Cartiglia ${ }^{a}$, R. Castello ${ }^{a, b}$, M. Costa ${ }^{a, b}$, N. Demaria ${ }^{a}$, A. Graziano ${ }^{a, b, 1}$, C. Mariotti $^{a}$,

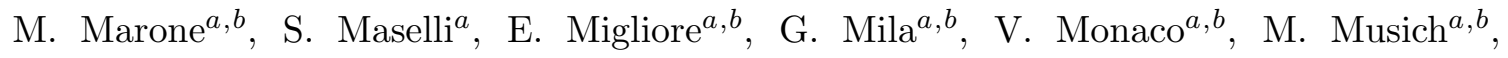


M.M. Obertino ${ }^{a, c}$, N. Pastrone ${ }^{a}$, M. Pelliccioni ${ }^{a, b}$, A. Potenza ${ }^{a, b}$, A. Romero ${ }^{a, b}$, M. Ruspa ${ }^{a, c}$, R. Sacchi ${ }^{a, b}$, V. Sola ${ }^{a, b}$, A. Solano ${ }^{a, b}$, A. Staiano ${ }^{a}$, A. Vilela Pereira ${ }^{a}$

INFN Sezione di Trieste ${ }^{a}$, Università di Trieste ${ }^{b}$, Trieste, Italy

S. Belforte ${ }^{a}$, F. Cossutti ${ }^{a}$, G. Della Ricca ${ }^{a, b}$, B. Gobbo ${ }^{a}$, D. Montanino ${ }^{a, b}$, A. Penzo $^{a}$

Kangwon National University, Chunchon, Korea

S.G. Heo, S.K. Nam

Kyungpook National University, Daegu, Korea

S. Chang, J. Chung, D.H. Kim, G.N. Kim, J.E. Kim, D.J. Kong, H. Park, S.R. Ro, D. Son, D.C. Son, T. Son

Chonnam National University, Institute for Universe and Elementary Particles, Kwangju, Korea

Zero Kim, J.Y. Kim, S. Song

Korea University, Seoul, Korea

S. Choi, B. Hong, M. Jo, H. Kim, J.H. Kim, T.J. Kim, K.S. Lee, D.H. Moon, S.K. Park, K.S. Sim

University of Seoul, Seoul, Korea

M. Choi, S. Kang, H. Kim, C. Park, I.C. Park, S. Park, G. Ryu

Sungkyunkwan University, Suwon, Korea

Y. Choi, Y.K. Choi, J. Goh, M.S. Kim, E. Kwon, J. Lee, S. Lee, H. Seo, I. Yu

Vilnius University, Vilnius, Lithuania

M.J. Bilinskas, I. Grigelionis, M. Janulis, D. Martisiute, P. Petrov, T. Sabonis

Centro de Investigacion y de Estudios Avanzados del IPN, Mexico City, Mexico

H. Castilla-Valdez, E. De La Cruz-Burelo, I. Heredia-de La Cruz, R. Lopez-Fernandez,

R. Magaña Villalba, A. Sánchez-Hernández, L.M. Villasenor-Cendejas

Universidad Iberoamericana, Mexico City, Mexico

S. Carrillo Moreno, F. Vazquez Valencia

Benemerita Universidad Autonoma de Puebla, Puebla, Mexico

H.A. Salazar Ibarguen

Universidad Autónoma de San Luis Potosí, San Luis Potosí, Mexico

E. Casimiro Linares, A. Morelos Pineda, M.A. Reyes-Santos

University of Auckland, Auckland, New Zealand

D. Krofcheck, J. Tam

University of Canterbury, Christchurch, New Zealand

P.H. Butler, R. Doesburg, H. Silverwood

National Centre for Physics, Quaid-I-Azam University, Islamabad, Pakistan

M. Ahmad, I. Ahmed, M.I. Asghar, H.R. Hoorani, W.A. Khan, T. Khurshid, S. Qazi 
Institute of Experimental Physics, Faculty of Physics, University of Warsaw, Warsaw, Poland

G. Brona, M. Cwiok, W. Dominik, K. Doroba, A. Kalinowski, M. Konecki, J. Krolikowski

Soltan Institute for Nuclear Studies, Warsaw, Poland

T. Frueboes, R. Gokieli, M. Górski, M. Kazana, K. Nawrocki, K. Romanowska-Rybinska, M. Szleper, G. Wrochna, P. Zalewski

Laboratório de Instrumentação e Física Experimental de Partículas, Lisboa, Portugal

N. Almeida, P. Bargassa, A. David, P. Faccioli, P.G. Ferreira Parracho, M. Gallinaro, P. Musella, A. Nayak, J. Pela ${ }^{1}$, P.Q. Ribeiro, J. Seixas, J. Varela

Joint Institute for Nuclear Research, Dubna, Russia

S. Afanasiev, I. Belotelov, P. Bunin, I. Golutvin, V. Karjavin, G. Kozlov, A. Lanev,

P. Moisenz, V. Palichik, V. Perelygin, M. Savina, S. Shmatov, V. Smirnov, A. Volodko,

A. Zarubin

Petersburg Nuclear Physics Institute, Gatchina (St Petersburg), Russia

V. Golovtsov, Y. Ivanov, V. Kim, P. Levchenko, V. Murzin, V. Oreshkin, I. Smirnov, V. Sulimov, L. Uvarov, S. Vavilov, A. Vorobyev, An. Vorobyev

Institute for Nuclear Research, Moscow, Russia

Yu. Andreev, A. Dermenev, S. Gninenko, N. Golubev, M. Kirsanov, N. Krasnikov, V. Matveev, A. Pashenkov, A. Toropin, S. Troitsky

Institute for Theoretical and Experimental Physics, Moscow, Russia

V. Epshteyn, V. Gavrilov, V. Kaftanov ${ }^{\dagger}$, M. Kossov ${ }^{1}$, A. Krokhotin, N. Lychkovskaya, V. Popov, G. Safronov, S. Semenov, V. Stolin, E. Vlasov, A. Zhokin

Moscow State University, Moscow, Russia

E. Boos, M. Dubinin ${ }^{23}$, L. Dudko, A. Ershov, A. Gribushin, O. Kodolova, I. Lokhtin, A. Markina, S. Obraztsov, M. Perfilov, S. Petrushanko, L. Sarycheva, V. Savrin, A. Snigirev P.N. Lebedev Physical Institute, Moscow, Russia

V. Andreev, M. Azarkin, I. Dremin, M. Kirakosyan, A. Leonidov, S.V. Rusakov, A. Vinogradov

State Research Center of Russian Federation, Institute for High Energy Physics, Protvino, Russia

I. Azhgirey, I. Bayshev, S. Bitioukov, V. Grishin ${ }^{1}$, V. Kachanov, D. Konstantinov, A. Korablev, V. Krychkine, V. Petrov, R. Ryutin, A. Sobol, L. Tourtchanovitch, S. Troshin, N. Tyurin, A. Uzunian, A. Volkov

University of Belgrade, Faculty of Physics and Vinca Institute of Nuclear Sciences, Belgrade, Serbia

P. Adzic ${ }^{24}$, M. Djordjevic, D. Krpic ${ }^{24}$, J. Milosevic 
Centro de Investigaciones Energéticas Medioambientales y Tecnológicas (CIEMAT), Madrid, Spain

M. Aguilar-Benitez, J. Alcaraz Maestre, P. Arce, C. Battilana, E. Calvo, M. Cepeda, M. Cerrada, M. Chamizo Llatas, N. Colino, B. De La Cruz, A. Delgado Peris, C. Diez Pardos, D. Domínguez Vázquez, C. Fernandez Bedoya, J.P. Fernández Ramos, A. Ferrando, J. Flix, M.C. Fouz, P. Garcia-Abia, O. Gonzalez Lopez, S. Goy Lopez, J.M. Hernandez, M.I. Josa, G. Merino, J. Puerta Pelayo, I. Redondo, L. Romero, J. Santaolalla, M.S. Soares, C. Willmott

\section{Universidad Autónoma de Madrid, Madrid, Spain}

C. Albajar, G. Codispoti, J.F. de Trocóniz

\section{Universidad de Oviedo, Oviedo, Spain}

J. Cuevas, J. Fernandez Menendez, S. Folgueras, I. Gonzalez Caballero, L. Lloret Iglesias, J.M. Vizan Garcia

Instituto de Física de Cantabria (IFCA), CSIC-Universidad de Cantabria, Santander, Spain

J.A. Brochero Cifuentes, I.J. Cabrillo, A. Calderon, S.H. Chuang, J. Duarte Campderros, M. Felcini ${ }^{25}$, M. Fernandez, G. Gomez, J. Gonzalez Sanchez, C. Jorda, P. Lobelle Pardo, A. Lopez Virto, J. Marco, R. Marco, C. Martinez Rivero, F. Matorras, F.J. Munoz Sanchez, J. Piedra Gomez ${ }^{26}$, T. Rodrigo, A.Y. Rodríguez-Marrero, A. Ruiz-Jimeno, L. Scodellaro, M. Sobron Sanudo, I. Vila, R. Vilar Cortabitarte

\section{CERN, European Organization for Nuclear Research, Geneva, Switzerland}

D. Abbaneo, E. Auffray, G. Auzinger, P. Baillon, A.H. Ball, D. Barney, A.J. Bell ${ }^{27}$, D. Benedetti, C. Bernet ${ }^{3}$, W. Bialas, P. Bloch, A. Bocci, S. Bolognesi, M. Bona, H. Breuker, K. Bunkowski, T. Camporesi, G. Cerminara, T. Christiansen, J.A. Coarasa Perez, B. Curé, D. D'Enterria, A. De Roeck, S. Di Guida, N. Dupont-Sagorin, A. Elliott-Peisert, B. Frisch, W. Funk, A. Gaddi, G. Georgiou, H. Gerwig, D. Gigi, K. Gill, D. Giordano, F. Glege, R. Gomez-Reino Garrido, M. Gouzevitch, P. Govoni, S. Gowdy, L. Guiducci, M. Hansen, C. Hartl, J. Harvey, J. Hegeman, B. Hegner, H.F. Hoffmann, A. Honma, V. Innocente, P. Janot, K. Kaadze, E. Karavakis, P. Lecoq, C. Lourenço, T. Mäki, M. Malberti, L. Malgeri, M. Mannelli, L. Masetti, A. Maurisset, F. Meijers, S. Mersi, E. Meschi, R. Moser, M.U. Mozer, M. Mulders, E. Nesvold ${ }^{1}$, M. Nguyen, T. Orimoto, L. Orsini, E. Perez, A. Petrilli, A. Pfeiffer, M. Pierini, M. Pimiä, D. Piparo, G. Polese, A. Racz, J. Rodrigues Antunes, G. Rolandi ${ }^{28}$, T. Rommerskirchen, M. Rovere, H. Sakulin, C. Schäfer, C. Schwick, I. Segoni, A. Sharma, P. Siegrist, M. Simon, P. Sphicas ${ }^{29}$, M. Spiropulu ${ }^{23}$, M. Stoye, P. Tropea, A. Tsirou, P. Vichoudis, M. Voutilainen, W.D. Zeuner

\section{Paul Scherrer Institut, Villigen, Switzerland}

W. Bertl, K. Deiters, W. Erdmann, K. Gabathuler, R. Horisberger, Q. Ingram, H.C. Kaestli, S. König, D. Kotlinski, U. Langenegger, F. Meier, D. Renker, T. Rohe, J. Sibille ${ }^{30}$, A. Starodumov ${ }^{31}$ 
Institute for Particle Physics, ETH Zurich, Zurich, Switzerland

L. Bäni, P. Bortignon, L. Caminada ${ }^{32}$, N. Chanon, Z. Chen, S. Cittolin, G. Dissertori, M. Dittmar, J. Eugster, K. Freudenreich, C. Grab, W. Hintz, P. Lecomte, W. Lustermann, C. Marchica ${ }^{32}$, P. Martinez Ruiz del Arbol, P. Milenovic ${ }^{33}$, F. Moortgat, C. Nägeli ${ }^{32}$, P. Nef, F. Nessi-Tedaldi, L. Pape, F. Pauss, T. Punz, A. Rizzi, F.J. Ronga, M. Rossini, L. Sala, A.K. Sanchez, M.-C. Sawley, B. Stieger, L. Tauscher ${ }^{\dagger}$, A. Thea, K. Theofilatos, D. Treille, C. Urscheler, R. Wallny, M. Weber, L. Wehrli, J. Weng

\section{Universität Zürich, Zurich, Switzerland}

E. Aguilo, C. Amsler, V. Chiochia, S. De Visscher, C. Favaro, M. Ivova Rikova, B. Millan Mejias, P. Otiougova, C. Regenfus, P. Robmann, A. Schmidt, H. Snoek

\section{National Central University, Chung-Li, Taiwan}

Y.H. Chang, K.H. Chen, C.M. Kuo, S.W. Li, W. Lin, Z.K. Liu, Y.J. Lu, D. Mekterovic, R. Volpe, J.H. Wu, S.S. Yu

National Taiwan University (NTU), Taipei, Taiwan

P. Bartalini, P. Chang, Y.H. Chang, Y.W. Chang, Y. Chao, K.F. Chen, W.-S. Hou, Y. Hsiung, K.Y. Kao, Y.J. Lei, R.-S. Lu, J.G. Shiu, Y.M. Tzeng, M. Wang

\section{Cukurova University, Adana, Turkey}

A. Adiguzel, M.N. Bakirci ${ }^{34}$, S. Cerci ${ }^{35}$, C. Dozen, I. Dumanoglu, E. Eskut, S. Girgis, G. Gokbulut, I. Hos, E.E. Kangal, A. Kayis Topaksu, G. Onengut, K. Ozdemir, S. Ozturk ${ }^{36}$, A. Polatoz, K. Sogut ${ }^{37}$, D. Sunar Cerci ${ }^{35}$, B. Tali ${ }^{35}$, H. Topakli ${ }^{34}$, D. Uzun, L.N. Vergili, M. Vergili

Middle East Technical University, Physics Department, Ankara, Turkey

I.V. Akin, T. Aliev, B. Bilin, S. Bilmis, M. Deniz, H. Gamsizkan, A.M. Guler, K. Ocalan, A. Ozpineci, M. Serin, R. Sever, U.E. Surat, E. Yildirim, M. Zeyrek

Bogazici University, Istanbul, Turkey

M. Deliomeroglu, D. Demir ${ }^{38}$, E. Gülmez, B. Isildak, M. Kaya ${ }^{39}$, O. Kaya ${ }^{39}$, M. Özbek, S. Ozkorucuklu ${ }^{40}$, N. Sonmez ${ }^{41}$

National Scientific Center, Kharkov Institute of Physics and Technology, Kharkov, Ukraine

L. Levchuk

\section{University of Bristol, Bristol, United Kingdom}

F. Bostock, J.J. Brooke, T.L. Cheng, E. Clement, D. Cussans, R. Frazier, J. Goldstein, M. Grimes, M. Hansen, D. Hartley, G.P. Heath, H.F. Heath, L. Kreczko, S. Metson, D.M. Newbold ${ }^{42}$, K. Nirunpong, A. Poll, S. Senkin, V.J. Smith, S. Ward

\section{Rutherford Appleton Laboratory, Didcot, United Kingdom}

L. Basso ${ }^{43}$, K.W. Bell, A. Belyaev ${ }^{43}$, C. Brew, R.M. Brown, B. Camanzi, D.J.A. Cockerill, J.A. Coughlan, K. Harder, S. Harper, J. Jackson, B.W. Kennedy, E. Olaiya, D. Petyt, B.C. Radburn-Smith, C.H. Shepherd-Themistocleous, I.R. Tomalin, W.J. Womersley, S.D. Worm 


\section{Imperial College, London, United Kingdom}

R. Bainbridge, G. Ball, J. Ballin, R. Beuselinck, O. Buchmuller, D. Colling, N. Cripps,

M. Cutajar, G. Davies, M. Della Negra, W. Ferguson, J. Fulcher, D. Futyan, A. Gilbert, A. Guneratne Bryer, G. Hall, Z. Hatherell, J. Hays, G. Iles, M. Jarvis, G. Karapostoli, L. Lyons, B.C. MacEvoy, A.-M. Magnan, J. Marrouche, B. Mathias, R. Nandi, J. Nash, A. Nikitenko ${ }^{31}$, A. Papageorgiou, M. Pesaresi, K. Petridis, M. Pioppi ${ }^{44}$, D.M. Raymond, S. Rogerson, N. Rompotis, A. Rose, M.J. Ryan, C. Seez, P. Sharp, A. Sparrow, A. Tapper, S. Tourneur, M. Vazquez Acosta, T. Virdee, S. Wakefield, N. Wardle, D. Wardrope, T. Whyntie

\section{Brunel University, Uxbridge, United Kingdom}

M. Barrett, M. Chadwick, J.E. Cole, P.R. Hobson, A. Khan, P. Kyberd, D. Leslie, W. Martin, I.D. Reid, L. Teodorescu

\section{Baylor University, Waco, USA}

K. Hatakeyama, H. Liu

The University of Alabama, Tuscaloosa, USA

C. Henderson

\section{Boston University, Boston, USA}

T. Bose, E. Carrera Jarrin, C. Fantasia, A. Heister, J. St. John, P. Lawson, D. Lazic, J. Rohlf, D. Sperka, L. Sulak

\section{Brown University, Providence, USA}

A. Avetisyan, S. Bhattacharya, J.P. Chou, D. Cutts, A. Ferapontov, U. Heintz, S. Jabeen, G. Kukartsev, G. Landsberg, M. Luk, M. Narain, D. Nguyen, M. Segala, T. Sinthuprasith, T. Speer, K.V. Tsang

\section{University of California, Davis, Davis, USA}

R. Breedon, M. Calderon De La Barca Sanchez, S. Chauhan, M. Chertok, J. Conway, P.T. Cox, J. Dolen, R. Erbacher, E. Friis, W. Ko, A. Kopecky, R. Lander, H. Liu, S. Maruyama, T. Miceli, M. Nikolic, D. Pellett, J. Robles, S. Salur, T. Schwarz, M. Searle, J. Smith, M. Squires, M. Tripathi, R. Vasquez Sierra, C. Veelken

University of California, Los Angeles, Los Angeles, USA

V. Andreev, K. Arisaka, D. Cline, R. Cousins, A. Deisher, J. Duris, S. Erhan, C. Farrell, J. Hauser, M. Ignatenko, C. Jarvis, C. Plager, G. Rakness, P. Schlein ${ }^{\dagger}$, J. Tucker, V. Valuev

University of California, Riverside, Riverside, USA

J. Babb, A. Chandra, R. Clare, J. Ellison, J.W. Gary, F. Giordano, G. Hanson, G.Y. Jeng, S.C. Kao, F. Liu, H. Liu, O.R. Long, A. Luthra, H. Nguyen, B.C. Shen ${ }^{\dagger}$, R. Stringer, J. Sturdy, S. Sumowidagdo, R. Wilken, S. Wimpenny

\section{University of California, San Diego, La Jolla, USA}

W. Andrews, J.G. Branson, G.B. Cerati, D. Evans, F. Golf, A. Holzner, R. Kelley, M. Lebourgeois, J. Letts, B. Mangano, S. Padhi, C. Palmer, G. Petrucciani, H. Pi, M. Pieri, R. Ranieri, M. Sani, V. Sharma, S. Simon, E. Sudano, M. Tadel, Y. Tu, A. Vartak, S. Wasserbaech ${ }^{45}$, F. Würthwein, A. Yagil, J. Yoo 


\section{University of California, Santa Barbara, Santa Barbara, USA}

D. Barge, R. Bellan, C. Campagnari, M. D’Alfonso, T. Danielson, K. Flowers, P. Geffert, J. Incandela, C. Justus, P. Kalavase, S.A. Koay, D. Kovalskyi, V. Krutelyov, S. Lowette, N. Mccoll, V. Pavlunin, F. Rebassoo, J. Ribnik, J. Richman, R. Rossin, D. Stuart, W. To, J.R. Vlimant

\section{California Institute of Technology, Pasadena, USA}

A. Apresyan, A. Bornheim, J. Bunn, Y. Chen, M. Gataullin, Y. Ma, A. Mott, H.B. Newman, C. Rogan, K. Shin, V. Timciuc, P. Traczyk, J. Veverka, R. Wilkinson, Y. Yang, R.Y. Zhu

\section{Carnegie Mellon University, Pittsburgh, USA}

B. Akgun, R. Carroll, T. Ferguson, Y. Iiyama, D.W. Jang, S.Y. Jun, Y.F. Liu, M. Paulini, J. Russ, H. Vogel, I. Vorobiev

\section{University of Colorado at Boulder, Boulder, USA}

J.P. Cumalat, M.E. Dinardo, B.R. Drell, C.J. Edelmaier, W.T. Ford, A. Gaz, B. Heyburn, E. Luiggi Lopez, U. Nauenberg, J.G. Smith, K. Stenson, K.A. Ulmer, S.R. Wagner, S.L. Zang

\section{Cornell University, Ithaca, USA}

L. Agostino, J. Alexander, D. Cassel, A. Chatterjee, S. Das, N. Eggert, L.K. Gibbons, B. Heltsley, W. Hopkins, A. Khukhunaishvili, B. Kreis, G. Nicolas Kaufman, J.R. Patterson, D. Puigh, A. Ryd, E. Salvati, X. Shi, W. Sun, W.D. Teo, J. Thom, J. Thompson, J. Vaughan, Y. Weng, L. Winstrom, P. Wittich

\section{Fairfield University, Fairfield, USA}

A. Biselli, G. Cirino, D. Winn

\section{Fermi National Accelerator Laboratory, Batavia, USA}

S. Abdullin, M. Albrow, J. Anderson, G. Apollinari, M. Atac, J.A. Bakken, S. Banerjee, L.A.T. Bauerdick, A. Beretvas, J. Berryhill, P.C. Bhat, I. Bloch, F. Borcherding, K. Burkett, J.N. Butler, V. Chetluru, H.W.K. Cheung, F. Chlebana, S. Cihangir, W. Cooper, D.P. Eartly, V.D. Elvira, S. Esen, I. Fisk, J. Freeman, Y. Gao, E. Gottschalk, D. Green, K. Gunthoti, O. Gutsche, J. Hanlon, R.M. Harris, J. Hirschauer, B. Hooberman, H. Jensen, M. Johnson, U. Joshi, R. Khatiwada, B. Klima, K. Kousouris, S. Kunori, S. Kwan, C. Leonidopoulos, P. Limon, D. Lincoln, R. Lipton, J. Lykken, K. Maeshima, J.M. Marraffino, D. Mason, P. McBride, T. Miao, K. Mishra, S. Mrenna, Y. Musienko ${ }^{46}$, C. Newman-Holmes, V. O'Dell, R. Pordes, O. Prokofyev, N. Saoulidou, E. Sexton-Kennedy, S. Sharma, W.J. Spalding, L. Spiegel, P. Tan, L. Taylor, S. Tkaczyk, L. Uplegger, E.W. Vaandering, R. Vidal, J. Whitmore, W. Wu, F. Yang, F. Yumiceva, J.C. Yun

\section{University of Florida, Gainesville, USA}

D. Acosta, P. Avery, D. Bourilkov, M. Chen, M. De Gruttola, G.P. Di Giovanni, D. Dobur, A. Drozdetskiy, R.D. Field, M. Fisher, Y. Fu, I.K. Furic, J. Gartner, B. Kim, J. Konigsberg, A. Korytov, A. Kropivnitskaya, T. Kypreos, K. Matchev, G. Mitselmakher, L. Muniz, C. Prescott, R. Remington, M. Schmitt, B. Scurlock, P. Sellers, N. Skhirtladze, M. Snowball, D. Wang, J. Yelton, M. Zakaria 
Florida International University, Miami, USA

C. Ceron, V. Gaultney, L. Kramer, L.M. Lebolo, S. Linn, P. Markowitz, G. Martinez, D. Mesa, J.L. Rodriguez

\section{Florida State University, Tallahassee, USA}

T. Adams, A. Askew, J. Bochenek, J. Chen, B. Diamond, S.V. Gleyzer, J. Haas,

S. Hagopian, V. Hagopian, M. Jenkins, K.F. Johnson, H. Prosper, L. Quertenmont, S. Sekmen, V. Veeraraghavan

Florida Institute of Technology, Melbourne, USA

M.M. Baarmand, B. Dorney, S. Guragain, M. Hohlmann, H. Kalakhety, R. Ralich, I. Vodopiyanov

University of Illinois at Chicago (UIC), Chicago, USA

M.R. Adams, I.M. Anghel, L. Apanasevich, Y. Bai, V.E. Bazterra, R.R. Betts, J. Callner, R. Cavanaugh, C. Dragoiu, L. Gauthier, C.E. Gerber, D.J. Hofman, S. Khalatyan, G.J. Kunde ${ }^{47}$, F. Lacroix, M. Malek, C. O'Brien, C. Silkworth, C. Silvestre, A. Smoron, D. Strom, N. Varelas

\section{The University of Iowa, Iowa City, USA}

U. Akgun, E.A. Albayrak, B. Bilki, W. Clarida, F. Duru, C.K. Lae, E. McCliment, J.P. Merlo, H. Mermerkaya ${ }^{48}$, A. Mestvirishvili, A. Moeller, J. Nachtman, C.R. Newsom, E. Norbeck, J. Olson, Y. Onel, F. Ozok, S. Sen, J. Wetzel, T. Yetkin, K. Yi

\section{Johns Hopkins University, Baltimore, USA}

B.A. Barnett, B. Blumenfeld, A. Bonato, C. Eskew, D. Fehling, G. Giurgiu, A.V. Gritsan, Z.J. Guo, G. Hu, P. Maksimovic, S. Rappoccio, M. Swartz, N.V. Tran, A. Whitbeck

The University of Kansas, Lawrence, USA

P. Baringer, A. Bean, G. Benelli, O. Grachov, R.P. Kenny Iii, M. Murray, D. Noonan, S. Sanders, J.S. Wood, V. Zhukova

\section{Kansas State University, Manhattan, USA}

A.F. Barfuss, T. Bolton, I. Chakaberia, A. Ivanov, S. Khalil, M. Makouski, Y. Maravin, S. Shrestha, I. Svintradze, Z. Wan

\section{Lawrence Livermore National Laboratory, Livermore, USA}

J. Gronberg, D. Lange, D. Wright

University of Maryland, College Park, USA

A. Baden, M. Boutemeur, S.C. Eno, D. Ferencek, J.A. Gomez, N.J. Hadley, R.G. Kellogg, M. Kirn, Y. Lu, A.C. Mignerey, K. Rossato, P. Rumerio, F. Santanastasio, A. Skuja, J. Temple, M.B. Tonjes, S.C. Tonwar, E. Twedt

\section{Massachusetts Institute of Technology, Cambridge, USA}

B. Alver, G. Bauer, J. Bendavid, W. Busza, E. Butz, I.A. Cali, M. Chan, V. Dutta, P. Everaerts, G. Gomez Ceballos, M. Goncharov, K.A. Hahn, P. Harris, Y. Kim, M. Klute, Y.-J. Lee, W. Li, C. Loizides, P.D. Luckey, T. Ma, S. Nahn, C. Paus, D. Ralph, C. Roland, 
G. Roland, M. Rudolph, G.S.F. Stephans, F. Stöckli, K. Sumorok, K. Sung, E.A. Wenger, R. Wolf, S. Xie, M. Yang, Y. Yilmaz, A.S. Yoon, M. Zanetti

\section{University of Minnesota, Minneapolis, USA}

S.I. Cooper, P. Cushman, B. Dahmes, A. De Benedetti, P.R. Dudero, G. Franzoni, J. Haupt, K. Klapoetke, Y. Kubota, J. Mans, N. Pastika, V. Rekovic, R. Rusack, M. Sasseville, A. Singovsky, N. Tambe

\section{University of Mississippi, University, USA}

L.M. Cremaldi, R. Godang, R. Kroeger, L. Perera, R. Rahmat, D.A. Sanders, D. Summers

\section{University of Nebraska-Lincoln, Lincoln, USA}

K. Bloom, S. Bose, J. Butt, D.R. Claes, A. Dominguez, M. Eads, J. Keller, T. Kelly, I. Kravchenko, J. Lazo-Flores, H. Malbouisson, S. Malik, G.R. Snow

\section{State University of New York at Buffalo, Buffalo, USA}

U. Baur, A. Godshalk, I. Iashvili, S. Jain, A. Kharchilava, A. Kumar, S.P. Shipkowski, K. Smith

\section{Northeastern University, Boston, USA}

G. Alverson, E. Barberis, D. Baumgartel, O. Boeriu, M. Chasco, S. Reucroft, J. Swain, D. Trocino, D. Wood, J. Zhang

\section{Northwestern University, Evanston, USA}

A. Anastassov, A. Kubik, N. Odell, R.A. Ofierzynski, B. Pollack, A. Pozdnyakov, M. Schmitt, S. Stoynev, M. Velasco, S. Won

\section{University of Notre Dame, Notre Dame, USA}

L. Antonelli, D. Berry, A. Brinkerhoff, M. Hildreth, C. Jessop, D.J. Karmgard, J. Kolb, T. Kolberg, K. Lannon, W. Luo, S. Lynch, N. Marinelli, D.M. Morse, T. Pearson, R. Ruchti, J. Slaunwhite, N. Valls, M. Wayne, J. Ziegler

\section{The Ohio State University, Columbus, USA}

B. Bylsma, L.S. Durkin, J. Gu, C. Hill, P. Killewald, K. Kotov, T.Y. Ling, M. Rodenburg, G. Williams

\section{Princeton University, Princeton, USA}

N. Adam, E. Berry, P. Elmer, D. Gerbaudo, V. Halyo, P. Hebda, A. Hunt, J. Jones, E. Laird, D. Lopes Pegna, D. Marlow, T. Medvedeva, M. Mooney, J. Olsen, P. Piroué, X. Quan, H. Saka, D. Stickland, C. Tully, J.S. Werner, A. Zuranski

\section{University of Puerto Rico, Mayaguez, USA}

J.G. Acosta, X.T. Huang, A. Lopez, H. Mendez, S. Oliveros, J.E. Ramirez Vargas, A. Zatserklyaniy

\section{Purdue University, West Lafayette, USA}

E. Alagoz, V.E. Barnes, G. Bolla, L. Borrello, D. Bortoletto, M. De Mattia, A. Everett, A.F. Garfinkel, L. Gutay, Z. Hu, M. Jones, O. Koybasi, M. Kress, A.T. Laasanen, 
N. Leonardo, C. Liu, V. Maroussov, P. Merkel, D.H. Miller, N. Neumeister, I. Shipsey,

D. Silvers, A. Svyatkovskiy, H.D. Yoo, J. Zablocki, Y. Zheng

Purdue University Calumet, Hammond, USA

P. Jindal, N. Parashar

\section{Rice University, Houston, USA}

C. Boulahouache, K.M. Ecklund, F.J.M. Geurts, B.P. Padley, R. Redjimi, J. Roberts, J. Zabel

\section{University of Rochester, Rochester, USA}

B. Betchart, A. Bodek, Y.S. Chung, R. Covarelli, P. de Barbaro, R. Demina, Y. Eshaq,

H. Flacher, A. Garcia-Bellido, P. Goldenzweig, Y. Gotra, J. Han, A. Harel, D.C. Miner,

D. Orbaker, G. Petrillo, W. Sakumoto, D. Vishnevskiy, M. Zielinski

The Rockefeller University, New York, USA

A. Bhatti, R. Ciesielski, L. Demortier, K. Goulianos, G. Lungu, S. Malik, C. Mesropian, M. Yan

\section{Rutgers, the State University of New Jersey, Piscataway, USA}

O. Atramentov, A. Barker, D. Duggan, Y. Gershtein, R. Gray, E. Halkiadakis, D. Hidas,

D. Hits, A. Lath, S. Panwalkar, R. Patel, K. Rose, S. Schnetzer, S. Somalwar, R. Stone,

S. Thomas

University of Tennessee, Knoxville, USA

G. Cerizza, M. Hollingsworth, S. Spanier, Z.C. Yang, A. York

Texas A\&M University, College Station, USA

R. Eusebi, W. Flanagan, J. Gilmore, A. Gurrola, T. Kamon, V. Khotilovich, R. Montalvo, I. Osipenkov, Y. Pakhotin, J. Pivarski, A. Safonov, S. Sengupta, A. Tatarinov, D. Toback, M. Weinberger

\section{Texas Tech University, Lubbock, USA}

N. Akchurin, C. Bardak, J. Damgov, C. Jeong, K. Kovitanggoon, S.W. Lee, T. Libeiro, P. Mane, Y. Roh, A. Sill, I. Volobouev, R. Wigmans, E. Yazgan

\section{Vanderbilt University, Nashville, USA}

E. Appelt, E. Brownson, D. Engh, C. Florez, W. Gabella, M. Issah, W. Johns, P. Kurt, C. Maguire, A. Melo, P. Sheldon, B. Snook, S. Tuo, J. Velkovska

University of Virginia, Charlottesville, USA

M.W. Arenton, M. Balazs, S. Boutle, B. Cox, B. Francis, R. Hirosky, A. Ledovskoy, C. Lin, C. Neu, R. Yohay

\section{Wayne State University, Detroit, USA}

S. Gollapinni, R. Harr, P.E. Karchin, P. Lamichhane, M. Mattson, C. Milstène, A. Sakharov

\section{University of Wisconsin, Madison, USA}

M. Anderson, M. Bachtis, J.N. Bellinger, D. Carlsmith, S. Dasu, J. Efron, K. Flood, L. Gray, K.S. Grogg, M. Grothe, R. Hall-Wilton, M. Herndon, A. Hervé, P. Klabbers, J. Klukas, A. Lanaro, C. Lazaridis, J. Leonard, R. Loveless, A. Mohapatra, F. Palmonari, D. Reeder, I. Ross, A. Savin, W.H. Smith, J. Swanson, M. Weinberg 
$\dagger$ : Deceased

1: Also at CERN, European Organization for Nuclear Research, Geneva, Switzerland

2: Also at Universidade Federal do ABC, Santo Andre, Brazil

3: Also at Laboratoire Leprince-Ringuet, Ecole Polytechnique, IN2P3-CNRS, Palaiseau, France

4: Also at Suez Canal University, Suez, Egypt

5: Also at British University, Cairo, Egypt

6: Also at Fayoum University, El-Fayoum, Egypt

7: Also at Soltan Institute for Nuclear Studies, Warsaw, Poland

8: Also at Massachusetts Institute of Technology, Cambridge, USA

9: Also at Université de Haute-Alsace, Mulhouse, France

10: Also at Brandenburg University of Technology, Cottbus, Germany

11: Also at Moscow State University, Moscow, Russia

12: Also at Institute of Nuclear Research ATOMKI, Debrecen, Hungary

13: Also at Eötvös Loránd University, Budapest, Hungary

14: Also at Tata Institute of Fundamental Research - HECR, Mumbai, India

15: Also at University of Visva-Bharati, Santiniketan, India

16: Also at Sharif University of Technology, Tehran, Iran

17: Also at Shiraz University, Shiraz, Iran

18: Also at Isfahan University of Technology, Isfahan, Iran

19: Also at Facoltà Ingegneria Università di Roma "La Sapienza", Roma, Italy

20: Also at Università della Basilicata, Potenza, Italy

21: Also at Laboratori Nazionali di Legnaro dell' INFN, Legnaro, Italy

22: Also at Università degli studi di Siena, Siena, Italy

23: Also at California Institute of Technology, Pasadena, USA

24: Also at Faculty of Physics of University of Belgrade, Belgrade, Serbia

25: Also at University of California, Los Angeles, Los Angeles, USA

26: Also at University of Florida, Gainesville, USA

27: Also at Université de Genève, Geneva, Switzerland

28: Also at Scuola Normale e Sezione dell' INFN, Pisa, Italy

29: Also at University of Athens, Athens, Greece

30: Also at The University of Kansas, Lawrence, USA

31: Also at Institute for Theoretical and Experimental Physics, Moscow, Russia

32: Also at Paul Scherrer Institut, Villigen, Switzerland

33: Also at University of Belgrade, Faculty of Physics and Vinca Institute of Nuclear Sciences, Belgrade, Serbia

34: Also at Gaziosmanpasa University, Tokat, Turkey

35: Also at Adiyaman University, Adiyaman, Turkey

36: Also at The University of Iowa, Iowa City, USA

37: Also at Mersin University, Mersin, Turkey

38: Also at Izmir Institute of Technology, Izmir, Turkey

39: Also at Kafkas University, Kars, Turkey

40: Also at Suleyman Demirel University, Isparta, Turkey

41: Also at Ege University, Izmir, Turkey

42: Also at Rutherford Appleton Laboratory, Didcot, United Kingdom

43: Also at School of Physics and Astronomy, University of Southampton, Southampton, United Kingdom

44: Also at INFN Sezione di Perugia; Università di Perugia, Perugia, Italy

45: Also at Utah Valley University, Orem, USA

46: Also at Institute for Nuclear Research, Moscow, Russia

47: Also at Los Alamos National Laboratory, Los Alamos, USA

48: Also at Erzincan University, Erzincan, Turkey 